\title{
A Multi-Level, Mobile-Enabled Intervention to Promote Physical Activity in Older Adults in the Primary Care Setting (iCanFit 2.0): Protocol for a Cluster Randomized Controlled Trial
}

Y Alicia Hong ${ }^{1}$, PhD; Samuel N Forjuoh ${ }^{2}$, MD, DrPH; Marcia G Ory ${ }^{1}$, MPH, PhD; Michael D Reis ${ }^{2}$, MD; Huiyan Sang $^{3}, \mathrm{PhD}$

\footnotetext{
${ }^{1}$ School of Public Health, Texas A\&M University, College Station, TX, United States

${ }^{2}$ Department of Family Medicine, Baylor Scott and White Health, Temple, TX, United States

${ }^{3}$ Department of Statistics, Texas A\&M University, College Station, TX, United States
}

\section{Corresponding Author:}

Y Alicia Hong, PhD

School of Public Health

Texas A\&M University

1266 TAMU

College Station, TX, 77843

United States

Phone: 19794369343

Email: yhong@sph.tamhsc.edu

\section{Abstract}

Background: Most older adults do not adhere to the US Centers for Disease Control physical activity guidelines; their physical inactivity contributes to overweight and multiple chronic conditions. An urgent need exists for effective physical activity-promotion programs for the large number of older adults in the United States.

Objective: This study presents the development of the intervention and trial protocol of iCanFit 2.0, a multi-level, mobile-enabled, physical activity-promotion program developed for overweight older adults in primary care settings.

Methods: The iCanFit 2.0 program was developed based on our prior mHealth intervention programs, qualitative interviews with older patients in a primary care clinic, and iterative discussions with key stakeholders. We will test the efficacy of iCanFit 2.0 through a cluster randomized controlled trial in six pairs of primary care clinics.

Results: The proposed protocol received a high score in a National Institutes of Health review, but was not funded due to limited funding sources. We are seeking other funding sources to conduct the project.

Conclusions: The iCanFit 2.0 program is one of the first multi-level, mobile-enabled, physical activity-promotion programs for older adults in a primary care setting. The development process has actively involved older patients and other key stakeholders. The patients, primary care providers, health coaches, and family and friends were engaged in the program using a low-cost, off-the-shelf mobile tool. Such low-cost, multi-level programs can potentially address the high prevalence of physical inactivity in older adults.

(JMIR Res Protoc 2017;6(9):e183) doi: 10.2196/resprot.8220

\section{KEYWORDS}

iCanFit; older adults; mHealth intervention; intervention development; intervention trial; primary care

\section{Introduction}

The benefits of regular physical activity on the well-being of older adults are well established. Even small increases in physical activity at a population level could have far-reaching positive impacts on chronic diseases such as diabetes, cardiovascular diseases, and several cancers [1-3]. The US
Centers for Disease Control and Prevention and the American College of Sports Medicine have recommended 150 minutes of moderate-to-vigorous physical activity (MVPA) per week [4]; however, less than 5\% of American older adults adhere to the guideline [5-7]. Physical inactivity is associated with high prevalence of overweight (60\%) and chronic conditions (80\%) in this population [8]. As the elderly population continues to 
grow $-13 \%$ of the US population will be older than 65 years of age by 2020 [8] - an urgent need exists for physical activity-promotion programs that can reach a large population of older adults efficiently.

Increasing older adults' physical activity, especially MVPA, is challenging. Literature suggests that effective physical activity-promotion programs are those built upon social and behavioral theories and practices, extend beyond the individual-level factors, and incorporate social and health care support [9-11]. When promoting physical activity in older adults, multi-level intervention programs that promote physical activity through goal setting and tracking at the individual level, social support at the interpersonal level, continuous monitoring at the health care level, and positive social norms at the community level are more likely to have sustainable effects [10-13].

More than $67 \%$ of older Americans (65 years or older) use the Internet and $42 \%$ own a mobile phone [14]; the digital divide in older adults has narrowed in the past decade [15]. The high rates of owning mobile tools suggest feasibility of mobile-based physical activity interventions. Mobile-based programs can reach a large number of patients efficiently; such programs can be easily tailored to individual needs and integrated into the health care system where electronic health records (EHRs) have been widely adopted [16-18]. In fact, a growing number of mobile-based, physical activity-promotion programs have been developed and tested in older adults $[19,20]$. However, recent reviews of existing programs have shown inconclusive evidence. While some studies report significant efficacy, others suggest minimal benefits, especially compared to usual care [20,21]. These programs, which typically used simple texting or short message service (SMS) text messaging to deliver reminders, had only short-term effects [22,23]. Some programs have required participants to use newly developed websites or mobile apps, thus limiting the population reach [10,21]. Despite inconclusive evidence from mobile-based, physical activity-promotion programs, reviews of existing literature have revealed some common characteristics of successful physical activity programs. They typically (1) incorporate the patient as an active participant in goal setting and tracking, (2) are based on behavioral and socioecological theory, (3) emphasize problem solving and the use of social support, and (4) provide both proactive and follow-up support [3,10,24]. Literature also documents the importance of social support, especially support from friends and family in mobile-based, physical activity-promotion programs [25].

Older adults tend to have more trust in their primary care providers (PCPs), or general practitioners in some countries, compared to other populations [26]. American older adults see their PCPs at least every 6 months [27]. Thus, the primary care clinics provide an ideal setting for delivering physical activity-promotion programs to older adults. Many PCPs, however, do not counsel their patients for physical activity promotion; they either have to address other medical complaints raised by the patients or they see no necessity of bringing up physical activity in the consultation with patients [28]. Our recent interviews with PCPs found that most physicians assumed patients understood the importance of physical activity and the lack of regular physical activity was due to patients' insufficient motivation [29,30]. A recent BMJ systematic review identified only 15 trials conducted in primary care organizations and the most typical intervention was a one-time simple counseling session by a PCP or nurse [13]. More research is therefore needed to explore the efficacy of theory-guided, physical activity-promotion programs in primary care settings.

We aim to address the literature gaps noted above by proposing a multi-level, mobile-enabled, physical activity-promotion program called iCanFit 2.0 in a primary care setting. Guided by socioecological theory, the iCanFit 2.0 program incorporates PCPs and health coaches in behavioral goal setting and continuous support for the patients. The intervention will exert effects at the individual, interpersonal, health care, and community levels. Mobile tools will facilitate patient-provider communication, enhance motivation, and provide ongoing feedback and social support to promote physical activity, as shown in Figure 1. To test the efficacy of iCanFit 2.0, we also designed a cluster randomized controlled trial (RCT) in a large health care organization.

\section{Methods}

\section{Development of iCanFit 2.0}

\section{Overview}

The development of the iCanFit 2.0 program was based on the following data sources and processes: (1) Preliminary studies with older adults in primary care settings and mHealth interventions including the iCanFit Web app, (2) qualitative interviews with older patients in a primary care clinic prior to this design, and (3) iterative discussions with stakeholders.

\section{Preliminary Studies}

From 2011 to 2015, we conducted the following formative research and mHealth interventions on physical activity promotion among older adults:

1. Assessment of overweight patients' barriers to physical activity from the perspectives of PCPs. Through online surveys with 57 PCPs and focus groups with 49 PCPs, we learned that PCPs were aware of the importance of counseling older patients regarding physical activity and identified lack of motivation and social support as major barriers to regular physical activity [28-30].

2. Use of the iPod Touch for patient health behavior assessment and patient-provider communication. We developed an app on the iPod Touch-before the iPad was released - so patients could complete a brief health behavior assessment (HBA) on a touch screen while waiting for appointments with a PCP. A colorful chart report was generated instantly (see Multimedia Appendix 1). When the patient walked into the appointment with a PCP, the report became a natural conversation starter and facilitated patient-provider communication and collaborative goal setting. We piloted this app with 109 patients in a primary care clinic and the results showed that $30 \%$ of the participants reported that their PCP discussed the report with them, $24 \%$ established behavioral goals with him or her as a result of the discussion, and $90 \%$ related positive 
experiences with using mobile tools to generate an HBA report [31].

3. Development and testing of the iCanFit interactive website for older adults. With the goal to promote physical activity in older cancer survivors, we developed an interactive website called iCanFit. We conducted three phases of research: formative research with key stakeholders [32], usability testing of the website with target users [33], and an efficacy trial with older cancer survivors [34]. The users of iCanFit reported high levels of usefulness and satisfaction. Participants reported a higher level of quality of life (effect size $=0.35$ ) and a higher level of physical activity (effect size $=0.45$ ) following the use of the $\mathrm{iCanFit}$ Web app [34].

\section{Qualitative Interviews With Older Diabetes Patients in a Primary Care Clinic}

The initial design of iCanFit 2.0 was the combination of the HBA tablet app and the interactive iCanFit Web app. As the initial design of iCanFit 2.0 evolved, we conducted qualitative interviews with 103 older diabetes patients in a primary care clinic. Considering most older adults have multiple chronic conditions, our preliminary studies focused on different chronic conditions to ensure that iCanFit 2.0 can serve the needs of a large number of older adults. The mean age of the participants in the qualitative interviews was 50 years and the mean years of living with diabetes was 10 years. Most of these older patients used the Internet and more than half had a mobile phone. They had positive attitudes toward a mobile-based, physical activity-promotion program and offered many suggestions and concerns for the design of iCanFit 2.0.

\section{Iterative Discussions With Key Stakeholders to Refine the Design}

After 10 years working in healthy aging and chronic disease management, we have established rapport with local communities and health care organizations; we have also always engaged key stakeholders in the intervention design, implementation, and evaluation. In designing iCanFit 2.0, we had a series of group discussions with our key stakeholders. We brought our initial design to the meeting and obtained their feedback; the iterative process continued until a satisfactory protocol was agreed upon by all key stakeholders. The current design of iCanFit 2.0 reflects the inputs from our target patients and other key stakeholders and is substantially different from the original design.

\section{Community Engagement}

Prior to completion of protocol design and project implementation, a Community Advisory Board (CAB) will be established, consisting of older patients, community leaders, health care providers, and administrators. At least five members of the $\mathrm{CAB}$ will be older patients. The $\mathrm{CAB}$ will have 10 members and vote on a director and a secretary. The CAB will meet with the project team every month in the first 6 months of the project and every 6 months afterward. The CAB will offer gatekeeper and stakeholder concerns as well as recommendations on program design, feasibility issues, implementation, and evaluation strategies; it will also help the research team interpret findings and advise on how to translate research findings into sustainable programs.

\section{Intervention Trial}

\section{Overview}

The intervention trial compares the effectiveness of the iCanFit 2.0 intervention program with a comparator program among overweight older adults in a primary care setting. To achieve this goal, we will conduct a cluster RCT in 12 family medicine clinics (six pairs) in Central Texas, USA. In each pair of comparable clinics, one will be randomized to the intervention group and one to the control group (see Figure 2). 
Figure 1. Theoretical framework of the multi-level intervention, iCanFit 2.0.

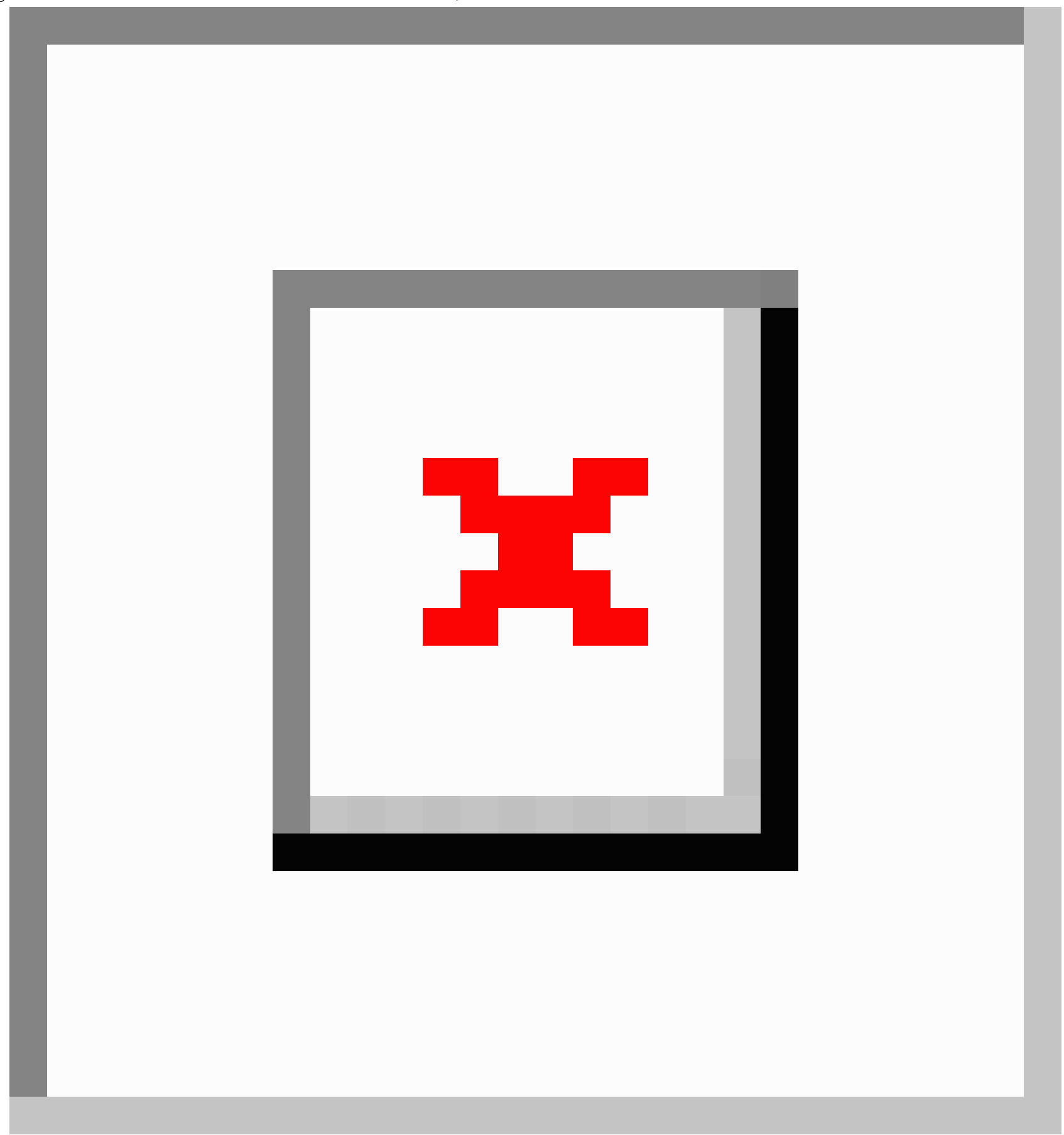


Figure 2. Intervention trial flowchart.

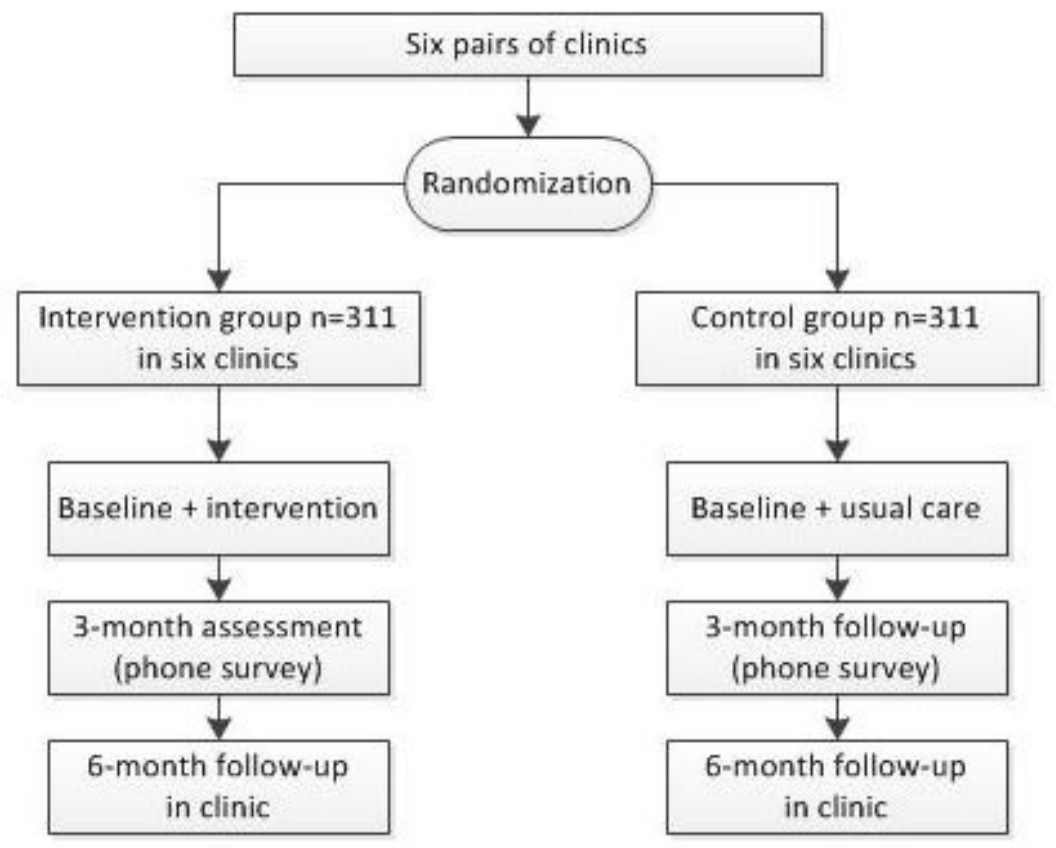

\section{Study Sites and Sources of Patients}

We will conduct the proposed trial in 12 family medicine clinics that belong to a large university-affiliated, integrated health care system in Central Texas. These clinics were selected because of their similarity in patient demographics, clinic operation, and service coverage. The 12 clinics will be grouped into six pairs. Clinics in each pair have been selected to have similar characteristics of size (ie, number of patient visits per year) and number of overweight older adults as well as situated miles apart to reduce possible contamination of the intervention. We will use a randomization table to assign the two clinics in each pair into the intervention group or the control group.

\section{iCanFit 2.0 Intervention Protocol}

As illustrated in Figure 3, the iCanFit 2.0 intervention protocol includes four steps. First, after the eligible participants complete the informed consent forms, they will complete an HBA on an iPad (see Multimedia Appendix 1 for sample screenshots), which takes about 15 minutes. Right after completion, patients will receive a printed copy of the HBA summary report with colorful charts of their current level of physical activity compared to the recommended physical activity levels (see sample summary reports in Multimedia Appendix 1). Meanwhile, the same report will be printed out in the office of the PCP with whom the patient has an appointment. Second, the patient brings the HBA report into his/her appointment with the PCP and the report serves as a natural conversation starter to facilitate patient-provider communication and joint goal setting. Third, right after the patient's appointment with the
PCP in the primary care clinic, a health coach will meet with the patient, further explain the HBA report, and ensure the patient has set a long-term physical activity goal, if the patient has not set one with the PCP. The health coach will give the patient a Fitbit Flex 2 (Fitbit Inc) (see Multimedia Appendix 2 and section below about the device) and demonstrate how to use the device. The health coach will also help the patient to create an account on the iCanFit online community. The health coach will advise on how to set short-term (eg, weekly) goals, track and sync data, share progress with family and friends via Facebook, and obtain personalized feedback on the iCanFit online community. The counseling session will last 30 minutes; a photo-illustrative brochure with instructions on how to use the Fitbit as well as account information and reminders will be given to the patient. Fourth, the health coach will constantly monitor patients' use of the Fitbit and iCanFit online community. Patients will receive incentives (eg, online badges, virtual coins, and honor levels) for meeting physical activity goals and updating their progress. Any questions posted on the iCanFit online community will be answered by the health coach within 12 hours. If a patient is "idle" for 2 weeks, the health coach will call the patient to offer help and address the patient's barriers. Patients will also receive a brochure of safety tips (see Multimedia Appendix 3), which details possible adverse events during exercise and how to take action depending on the situation. For adverse events that need immediate medical attention, patients are advised to go to the nearest emergency room. For nonurgent matters, they can contact the health coach, who can assist with scheduling a clinic appointment with the patient's PCP. 
Figure 3. Process of iCanFit 2.0 intervention. PCP: primary care provider.

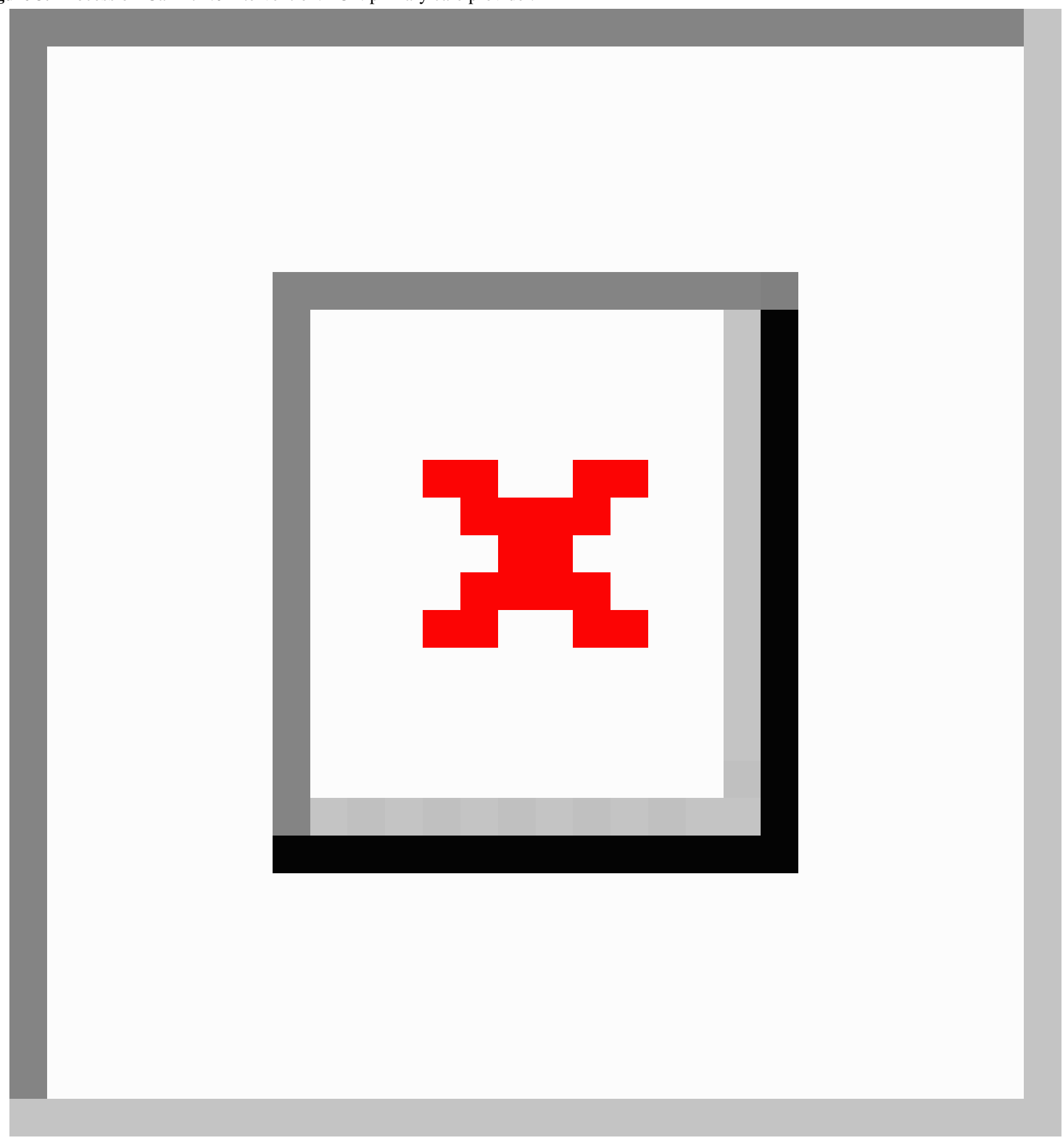

Figure 4. Process of comparator protocol (for patients in the control group). PCP: primary care provider.

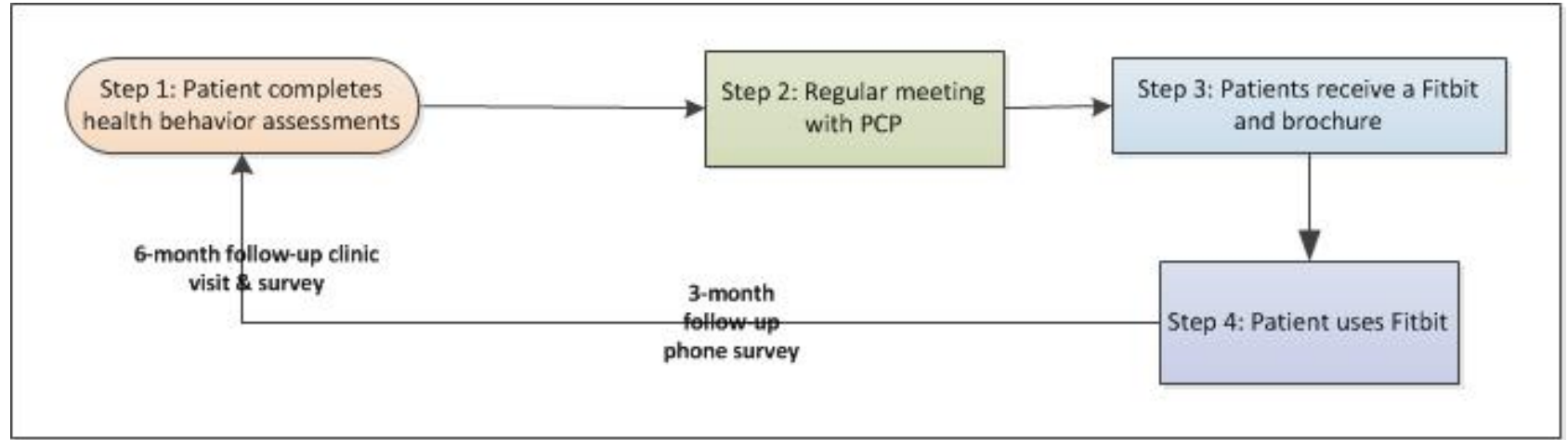




\section{Comparator}

The patients in the control group will receive usual care enhanced with a Fitbit Flex 2, as shown in Multimedia Appendix 2. The Fitbit Flex 2 was released in 2016, costs US \$75, and weighs a quarter of an ounce. It is compatible with iPhones and Android phones, only needs to charge once a week, syncs automatically with mobile phones within 20 feet, and easily syncs to a computer with a USB port. It tracks steps taken, stairs climbed, calories burned, distance travelled, and sleep time. Figure 4 illustrates the process of the comparator protocol. After eligible patients provide informed consent, they will first complete an HBA on an iPad while waiting for their appointment. Unlike patients in the intervention group, patients in the control group will not receive any reports from their HBA; after their appointment with the PCP, they will receive a Fitbit Flex 2 and a brochure explaining how to use the Fitbit and the benefits of regular physical activity. Patients in the control group will not be given any information about the iCanFit online community; neither will they receive counseling or monitoring from health coaches. Similar to the intervention group, control group patients will receive the safety tips of exercise brochure.

\section{Recruitment of Patients}

Eligibility of participants includes the following: (1) 60 years of age or older, (2) body mass index (BMI) of $25 \mathrm{~kg} / \mathrm{m}^{2}$ or higher, (3) have no medical condition prohibiting regular physical activity as shown in the EHR, (4) have access to the Internet through a computer or mobile phone, and (5) have a phone that can receive SMS text messages or phone calls. Based on our prior experience of recruiting participants for mHealth interventions in primary care settings and the consideration of minimal interruption of daily operation of the clinic, we will recruit participants as follows: in the EHR system, patients who are 60 years of age or older, have a BMI of $25 \mathrm{~kg} / \mathrm{m}^{2}$ or higher, and have no medical condition that prohibits them from exercise will be flagged. When a flagged patient checks in for his/her appointment, a health coach will be notified, who will approach the patient and explain the purpose of the project and check if the patient meets all the criteria. Eligible patients will be invited to participate in the study. Participation is completely voluntary; if the patient declines to participate, we will take notes regarding the patient's demographic information for future analysis. If the patient agrees to participate, she/he will complete the informed consent form and be able to begin the study at the very visit when they are recruited. In case some patients agree to participate but cannot start the study at that visit, we will schedule another visit for these patients to start the study within a month.

\section{Program Fidelity}

We will take the following measures to ensure the program is delivered with high quality and good fidelity. First, all research staff will receive 2 months of intensive, project-specific training on research ethics, intervention design, and project implementation. They will also receive training on interviewing patients and research conduct at the family medicine clinics where the intervention will take place. Health coaches will receive additional training on how to interact with patients, how to demonstrate the use of the Fitbit Flex 2, and how to set short-term goals and track progress on the iCanFit online community. They will practice health coaching with patient representatives from our $\mathrm{CAB}$ until satisfactory performance is demonstrated. We will also have refresher training once a year during the project implementation. Second, health coaches will counsel patients under the supervision of a registered nurse; an experienced nurse will randomly check these counseling sessions and provide timely feedback. As part of quality control, our CAB will conduct site visits quarterly throughout the study. Third, the principal investigators of the project will work in the participating clinics for quality assurance and address any issues that may come up during the trial.

\section{Patient Retention}

To ensure we retain most of the patients during the intervention trial, the following measures were proposed. First, we will explain to the participants the longitudinal nature of the study during the informed consent and the fact that they will have a phone survey in 3 months and a follow-up clinic visit in 6 months. Second, 2 weeks prior to the follow-up survey or visit, we will remind the participants via SMS text message or phone call and help them schedule the appointment at their convenience. Third, for patients who do not comply with the intervention protocol, for example, do not wear the Fitbit constantly or do not sync the data in a timely fashion, we will send friendly reminders via SMS text message and motivate them with positive outcomes of physical activity and social support from the iCanFit community. Patients who continuously ignore our reminders and invitations-for 2 consecutive weeks-will be considered dropouts. We expect a $20 \%$ attrition rate at 6 months. Fourth, some participants might lose their Fitbit device during the trial. We will immediately replace a Fitbit if lost. Based on our prior experiences [35,36], less than $5 \%$ of participants may lose the mobile device during the trial. Finally, some overweight older adults may report discomfort in exercise. During the counseling by the health coach at baseline, we will advise participants to slowly increase their physical activity level and to monitor their heart rates. All participants will have a brochure of safety tips (see Multimedia Appendix 3) outlining typical adverse events and how to take appropriate actions in case of such an event. The patient is advised to go to the nearest emergency room for an event that needs immediate care. For nonurgent issues that require consultation with a PCP, the health coach will assist to schedule an appointment.

\section{Outcome Evaluation}

\section{Outcome Measures and Sources of Data}

The evaluation of the iCanFit, physical activity-promotion program will be based on three datasets:

1. HBA surveys collected using the iPad at baseline and two follow-up surveys. As shown in Table 1, the survey includes demographic information, technology use and eHealth literacy [37], current level of physical activity [38], quality of life [39], patient-provider communication [40], perceived support from the health care team [41], and perceived social support for physical activity from the community [42]. All of these measures are based on validated scales with good 
validity and reliability. The first follow-up is a phone survey 3 months after baseline and the second follow-up is a clinic visit 6 months after baseline. This arrangement is based on the consideration that all overweight older adults are asked to visit their PCP at least every 6 months [27].

2. Fitbit data. Two types of Fitbit data will be collected for evaluation: physical activity data recorded on Fitbit and patient interactions with the health coaches and peers in the iCanFit online community.

3. Clinic data. We will collect patients' weight and adverse events related to participating in the study from the EHRs at the clinic visits at baseline and at the 6-month follow-up.

\section{Power and Sample Size Calculations}

The primary outcome of the study is the total minutes of MVPA per week. MVPA is measured as "very active" and "fairly active" on Fitbit. The secondary outcome of the study is the patient's self-report quality of life, measured by the 12-Item Short Form Survey [39]. We hypothesize the effect size of the iCanFit 2.0 to be 0.25 , based on a prior Fitbit intervention trial [43]. Following the procedure developed by Cohen, the calculation of sample size is carried out in three steps by assuming a .05 significance level to achieve the power of 0.8 [44]. First, we assume that two independent samples can be obtained for the same size $n_{1}$ each. Then the total sample size is $n=2 \times n_{1}$. In this ideal scenario, the total sample size $n=398$ is required to detect the effect size $d=0.25$ between the two population means. Second, because the randomization occurs at the clinic level, we need to consider the clustering effect. Previous similar research suggests a small clustering effect due to multiple patients per physician, but virtually no clustering effect between patients with different physicians in the same clinic [45]. We assume that if the intraclass correlation coefficient (ICC) per patient is equal to .05 , and that ICC per physician is equal to 0 , then equation 1 holds:

$$
\begin{aligned}
& \text { ICC }=(1+\text { ICC_patient }) \times(1+\text { ICC_physican })-1= \\
& .05 \text { (1) }
\end{aligned}
$$

Thus, the design effect is as follows in equation 2:

$$
\mathrm{D}=1+(\mathrm{m}-1) \times \operatorname{ICC}(2)
$$

where $m$ is the average number of patients per physician in our effective sample. If we restrict $m$ to be no more than 6 , then $\mathrm{D}=1+5 \times .05=1.25$. We would need a sample size of 498 (ie, $398 \times 1.25)$ after factoring in cluster effects. Third, based on our prior study of RCT primary care settings [36], we assume the attrition rate to be 0.2 after 12 months, thus the required sample size at baseline is 622 in total (ie, 498/[1-0.2]), or 52 patients per clinic on average. We will recruit no fewer than 30 and no more than 70 patients from each participating clinic.

\section{Data Analysis Plan}

Our analysis has been planned to correspond to the study's main aim. We will begin with exploratory data analyses. Demographic and baseline characteristics for the participants will be summarized using descriptive statistics overall and by intervention and control groups to assess baseline comparability.
Prior to analyses, we will check continuous outcome distributions and apply normalizing transformation where needed.

Our main goal is to test the hypothesis that patients in the intervention group will have more MVPA per week than the control group. To test this hypothesis, we will compare major outcomes between the intervention and control groups. We will implement multi-level regression models (eg, hierarchical linear models and mixed-effect models). Multi-level regression models are needed to account for an ICC that results from clinic-level observations [45]. Patients' observations over time are nested within PCPs, and PCPs are nested within clinics.

Equation 3 presents an example of a multi-level regression model for a continuous outcome $\mathrm{Y}_{\mathrm{ijt}}$, on clinic $i, \operatorname{PCP} j$, and time point $t$. Our analyses will also include background characteristics that are not included in equation 3 for the sake of brevity. Two predictors, time (TIME) and intervention group (INTV), are classified as $1=\mathrm{iCanFit}$ and $0=$ control. The multi-level regression model is given as follows:

$$
\begin{aligned}
& \mathrm{Y}_{\mathrm{ijt}}=\alpha_{0}+\mathrm{INTV}_{\mathrm{i}} \times \alpha_{1}+\text { TIME }_{\mathrm{ijt}} \times \alpha_{2}+\mathrm{INTV}_{\mathrm{i}} \\
& \mathrm{X} \mathrm{TIME}_{\mathrm{ijt}} \times \alpha_{3}+\zeta_{\mathrm{ij}}+\varepsilon_{\mathrm{ijt}} \text { (3) }
\end{aligned}
$$

where $\alpha_{0}, \alpha_{1}, \alpha_{2}$, and $\alpha_{3}$ are the fixed effects, $\zeta_{\mathrm{ij}}$ is a random effect (ie, random intercept) for each PCP in each clinic, and $\varepsilon_{\mathrm{ijt}}$ is the residual error for repeated observations over time. The random effect is assumed to be normally distributed with mean zero. The residual error is assumed to be multivariate normally distributed across repeated observations, with mean zero and a covariance matrix that models the autocorrelation among repeated observations [46]. We will examine model fit statistics to choose an appropriate covariance structure. Hypothesis testing will be carried out to test for intervention effects on the outcome of interest over time. Referring to equation 3, this is equivalent to testing the hypothesis $\mathrm{H}_{0}: \alpha_{3}=0$. If higher outcome values are desirable, then a positive significant $\alpha_{3}$ parameter indicates a positive intervention effect (ie, we reject $\mathrm{H}_{0}: \alpha_{3}=0$ ). We will use the SAS version 9.0 (SAS Institute Inc) PROC MIXED procedure and PROC GLIMMIX procedure to fit multi-level models to continuous and binary data, respectively.

We will also apply structural equation modeling (SEM) to examine the extent to which the intervention takes effect at individual, interpersonal, health care, and community levels as shown in Figure 1. Models will be constructed to measure direct and indirect effects. We will also analyze whether increased MVPA is a mediator for improved clinical outcomes. Latent variables are similar to random effects, accounting for nested observations. We will use Mplus version 3.1 (Muthén \& Muthén) to fit SEM for continuous and binary data $[47,48]$.

\section{Results}

The proposed protocol received a high score in a National Institutes of Health review, but was not funded due to limited funding sources. We are seeking other funding sources to conduct the project. 
Table 1. Data collected and instruments used in the survey.

\begin{tabular}{|c|c|c|c|}
\hline Domain & Scale or indicators & Data collection mode & $\begin{array}{l}\text { Number } \\
\text { of items }\end{array}$ \\
\hline Demographics & $\begin{array}{l}\text { Age, gender, race/ethnicity, marital status, education, income, } \\
\text { insurance, chronic conditions, and perceived health }\end{array}$ & Survey with patients at baseline & 11 \\
\hline Technology use & eHealth literacy scale (E-HEALS), alpha=.78 [37] & Survey with patients at baseline & 11 \\
\hline Physical activity & International Physical Activity Questionnaire, alpha=.76 [38] & $\begin{array}{l}\text { Surveys with patients at baseline } \\
\text { and follow-up }\end{array}$ & 4 \\
\hline Quality of life (primary outcome) & 12-Item Short Form Survey, alpha=.81 [39] & $\begin{array}{l}\text { Surveys with patients at baseline } \\
\text { and follow-up }\end{array}$ & 12 \\
\hline Patient-physician communication & Provider Patient Communication Scale, alpha $=.80[40]$ & $\begin{array}{l}\text { Surveys with patients at baseline } \\
\text { and follow-up }\end{array}$ & 4 \\
\hline Support from health care team & $\begin{array}{l}\text { Patient Assessment of Chronic Illness Care (PACIC), alpha=.79 } \\
\text { [41] }\end{array}$ & $\begin{array}{l}\text { Surveys with patients at baseline } \\
\text { and follow-up }\end{array}$ & 11 \\
\hline Support from broader community & Perceived social support for diet and exercise, alpha=.78 [42] & $\begin{array}{l}\text { Surveys with patients at baseline } \\
\text { and follow-up }\end{array}$ & 9 \\
\hline Total minutes of exercise per week & Total minutes of moderate-to-vigorous physical activity a week & Fitbit & Varies \\
\hline Patient-provider communication & Number of questions sent by patient & iCanFit community & Varies \\
\hline Patient engagement & $\begin{array}{l}\text { Frequency of log-ins, syncs, and communication with iCanFit } \\
\text { community and health coach }\end{array}$ & $\begin{array}{l}\text { iCanFit community and follow- } \\
\text { up survey }\end{array}$ & Varies \\
\hline $\begin{array}{l}\text { Moderate-to-vigorous physical } \\
\text { activity (primary outcome) }\end{array}$ & Total minutes of moderate-to-vigorous physical activity a week & Fitbit & 1 \\
\hline $\begin{array}{l}\text { Overall experience with the iCan- } \\
\text { Fit program }\end{array}$ & $\begin{array}{l}\text { Experience of the intervention and follow-up, intention of contin- } \\
\text { uing use of Fitbit and iCanFit community, and suggestions for } \\
\text { improving the program }\end{array}$ & Follow-up survey & Varies \\
\hline Secondary clinic outcomes & $\begin{array}{l}\text { Weight, chronic condition, and number of sickness clinic visits } \\
\text { and adverse events }\end{array}$ & Medical records & 4 \\
\hline
\end{tabular}

\section{Discussion}

The iCanFit 2.0 intervention protocol has the following four strengths:

1. 1. It is a mobile-enabled, multi-level intervention. Most existing mobile-based, physical activity-promotion programs are individual oriented and expect users to change behaviors after using the mobile tool; the complex social environment for behavioral change and maintenance has not been addressed [5,10,25]. The iCanFit 2.0 involves patients, PCPs, health coaches, and family and friends throughout the process instead of simply targeting the patients alone, thus shifting the focus from just a mobile tool to using mobile tools to foster a social environment for behavioral change and maintenance.

2. 2. The intervention delivery is compatible with normal clinic operation. The implementation of our program is designed to fit the normal operation of the primary care clinics. For example, instead of recruiting eligible patients through mail or phone invitation [49], we will flag eligible participants in the EHR system; when these flagged patients come to visit their PCP for clinic appointments, they will be invited to participate in the study. While waiting for their appointments, patients will complete a brief HBA; immediately generated reports via the office Wi-Fi system will be sent to both patient and PCP for better patient-provider communication. Health coaches will use mobile tools for continuous monitoring, not only reducing the burden of the PCPs but also increasing service efficiency and patient outreach. Because the majority of older adults typically see their PCPs at least every 6 months [27], we set our follow-up clinic visit at 6 months. These implementation strategies will allow the proposed intervention to be sustainable and scalable.

3. 3. The program uses the low-cost, off-the-shelf mobile device, Fitbit, for older adults. Many existing mHealth interventions have typically used newly developed mobile apps, which resulted in limited scalability and sustainability of the program [19,21]. We chose to use the Fitbit Flex 2 because of its low cost, ease of use, high compatibility, and documented reliability and validity $[35,50,51]$.

4. 4. The participants in the control group also receive a Fitbit Flex 2, allowing us to test the intervention effect of iCanFit 2.0 versus the mobile device alone.

The iCanFit 2.0 program also has the following limitations:

1. 1. iCanFit 2.0 is a complex intervention with multiple components; it requires buy-in from the primary care clinics and especially the PCPs. It also requires skilled coordination and joint efforts by multiple parties.

2. 2. iCanFit 2.0 needs well-trained health coaches for counseling and monitoring of the patients.

3. 3. Some older patients may not have Internet access either via computers or mobile phones and, therefore, may not be able to join the study. 
4. 4. The control group does not have regular monitoring and social support and may suffer a higher rate of attrition.

5. 5. The iCanFit online community needs active users to maintain the positive social norm, which may be challenging for older adults. It merits further study on how to further engage older adults and obtain social support online.
Despite these limitations, to the best of our knowledge, the iCanFit 2.0 intervention will be one of the first multi-level, mobile-enabled, physical activity-promotion programs for older adults in a primary care setting. It was built upon our 10 years of research of a mobile-based intervention to promote physical activity in older adults. We have engaged patients and other key stakeholders throughout the design and will continue to do so in the intervention trial.

\section{Acknowledgments}

The development of iCanFit 2.0 was supported by an incentive fund from the Southwest Rural Health Research Center and the Texas A\&M University Program to Enhance Scholarly and Creative Activities (PESCA) award.

\section{Conflicts of Interest}

None declared.

\section{Multimedia Appendix 1}

Health behavior assessment screenshots and sample reports.

[PDF File (Adobe PDF File), 611KB-Multimedia Appendix 1]

\section{Multimedia Appendix 2}

Fitbit Flex 2 illustration and features.

[JPG File, 12KB-Multimedia Appendix 2]

\section{Multimedia Appendix 3}

Safety tips brochure.

\section{[PDF File (Adobe PDF File), 42KB-Multimedia Appendix 3]}

\section{References}

1. Sattelmair J, Pertman J, Ding EL, Kohl HW, Haskell W, Lee I. Dose response between physical activity and risk of coronary heart disease: A meta-analysis. Circulation 2011 Aug 16;124(7):789-795 [FREE Full text] [doi:

10.1161/CIRCULATIONAHA.110.010710] [Medline: 21810663]

2. Woodcock J, Franco OH, Orsini N, Roberts I. Non-vigorous physical activity and all-cause mortality: Systematic review and meta-analysis of cohort studies. Int J Epidemiol 2011 Feb;40(1):121-138. [doi: 10.1093/ije/dyq104] [Medline: 20630992]

3. Winzer B, Whiteman D, Reeves M, Paratz JD. Physical activity and cancer prevention: A systematic review of clinical trials. Cancer Causes Control 2011 Jun;22(6):811-826. [doi: 10.1007/s10552-011-9761-4] [Medline: 21461921]

4. American College of Sports Medicine. In: Pescatello LS, Arena R, Riebe D, Thompson PD, editors. ACSM's Guidelines for Exercise Testing and Prescription. 9th edition. Philadelphia, PA: Wolters Kluwer Health, Lippincott Williams \& Wilkins; 2014.

5. Hallal PC, Andersen LB, Bull FC, Guthold R, Haskell W, Ekelund U, Lancet Physical Activity Series Working Group. Global physical activity levels: Surveillance progress, pitfalls, and prospects. Lancet 2012 Jul 21;380(9838):247-257. [doi: 10.1016/S0140-6736(12)60646-1] [Medline: 22818937]

6. Moore LV, Harris CD, Carlson SA, Kruger J, Fulton JE. Trends in no leisure-time physical activity--United States, $1988-2010$. Res Q Exerc Sport 2012 Dec;83(4):587-591. [doi: 10.1080/02701367.2012.10599884] [Medline: 23367822]

7. Evenson KR, Buchner DM, Morland KB. Objective measurement of physical activity and sedentary behavior among US adults aged 60 years or older. Prev Chronic Dis 2012;9:E26 [FREE Full text] [Medline: 22172193]

8. Ward BW, Schiller JS. Prevalence of multiple chronic conditions among US adults: Estimates from the National Health Interview Survey, 2010. Prev Chronic Dis 2013 Apr 25;10:E65 [FREE Full text] [doi: 10.5888/pcd10.120203] [Medline: 23618545]

9. Hagger MS, Chatzisarantis NLD, Biddle SJH. A meta-analytic review of the theories of reasoned action and planned behavior in physical activity: Predictive validity and the contribution of additional variables. J Sport Exerc Psychol 2002;24(1):3-32 ISSN: ISSN-0895-2779. 
10. Heath G, Parra D, Sarmiento O, Andersen L, Owen N, Goenka S, Lancet Physical Activity Series Working Group. Evidence-based intervention in physical activity: Lessons from around the world. Lancet 2012 Jul 21;380(9838):272-281 [FREE Full text] [doi: 10.1016/S0140-6736(12)60816-2] [Medline: 22818939]

11. Cress ME, Buchner DM, Prohaska T, Rimmer J, Brown M, Macera C, et al. Best practices for physical activity programs and behavior counseling in older adult populations. J Aging Phys Act 2005 Jan;13(1):61-74. [Medline: 15677836]

12. Chodzko-Zajko WJ. Exercise and physical activity for older adults. Kinesiology Review 2014;3(1):101-106.

13. Orrow G, Kinmonth A, Sanderson S, Sutton S. Effectiveness of physical activity promotion based in primary care: Systematic review and meta-analysis of randomised controlled trials. BMJ 2012 Mar 26;344:e1389 [FREE Full text] [Medline: 22451477]

14. Anderson M, Perrin A. Pew Research Center. Washington, DC: Pew Internet \& American Life Project; 2017 May 17. Technology use among seniors URL: http://www.pewinternet.org/2017/05/17/technology-use-among-seniors/ [accessed 2017-06-15] [WebCite Cache ID 6rFdbcF8V]

15. Hong YA, Cho J. Has the digital health divide widened? Trends of health-related Internet use among older adults from 2003 to 2011. J Gerontol B Psychol Sci Soc Sci 2017 Sep 01;72(5):856-863. [doi: 10.1093/geronb/gbw100] [Medline: 27558403]

16. Baker LC, Johnson SJ, Macaulay D, Birnbaum H. Integrated telehealth and care management program for Medicare beneficiaries with chronic disease linked to savings. Health Aff (Millwood) 2011 Sep;30(9):1689-1697 [FREE Full text] [doi: 10.1377/hlthaff.2011.0216] [Medline: 21900660]

17. Satariano W, Scharlach A, Lindeman D. Aging, place, and technology: Toward improving access and wellness in older populations. J Aging Health 2014 Dec;26(8):1373-1389. [doi: 10.1177/0898264314543470] [Medline: 25502245]

18. Marcotte L, Seidman J, Trudel K, Berwick D, Blumenthal D, Mostashari F, et al. Achieving meaningful use of health information technology: A guide for physicians to the EHR incentive programs. Arch Intern Med 2012 May 14;172(9):731-736. [doi: 10.1001/archinternmed.2012.872] [Medline: 22782203]

19. Lyons E, Lewis Z, Mayrsohn B, Rowland JL. Behavior change techniques implemented in electronic lifestyle activity monitors: A systematic content analysis. J Med Internet Res 2014 Aug 15;16(8):e192 [FREE Full text] [doi: 10.2196/jmir.3469] [Medline: 25131661]

20. Stephens J, Allen J. Mobile phone interventions to increase physical activity and reduce weight: A systematic review. J Cardiovasc Nurs 2013;28(4):320-329 [FREE Full text] [doi: 10.1097/JCN.0b013e318250a3e7] [Medline: 22635061]

21. Fanning J, Mullen S, McAuley E. Increasing physical activity with mobile devices: A meta-analysis. J Med Internet Res 2012 Nov 21;14(6):e161 [FREE Full text] [doi: 10.2196/jmir.2171] [Medline: 23171838]

22. Blackman K, Zoellner J, Berrey L, Alexander R, Fanning J, Hill J, et al. Assessing the internal and external validity of mobile health physical activity promotion interventions: A systematic literature review using the RE-AIM framework. J Med Internet Res 2013 Oct 04;15(10):e224 [FREE Full text] [doi: 10.2196/jmir.2745] [Medline: 24095951]

23. Buchholz S, Wilbur J, Ingram D, Fogg L. Physical activity text messaging interventions in adults: A systematic review. Worldviews Evid Based Nurs 2013 Aug;10(3):163-173. [doi: 10.1111/wvn.12002] [Medline: 23746267]

24. Greaves CJ, Sheppard KE, Abraham C, Hardeman W, Roden M, Evans PH, IMAGE Study Group. Systematic review of reviews of intervention components associated with increased effectiveness in dietary and physical activity interventions. BMC Public Health 2011 Feb 18;11(1):119 [FREE Full text] [doi: 10.1186/1471-2458-11-119] [Medline: 21333011]

25. Bauman AE, Reis RS, Sallis JF, Wells JC, Loos RJF, Martin BW, Lancet Physical Activity Series Working Group. Correlates of physical activity: Why are some people physically active and others not? Lancet 2012 Jul 21;380(9838):258-271. [doi: 10.1016/S0140-6736(12)60735-1] [Medline: 22818938]

26. Costello E, Leone JE, Ellzy M, Miller TA. Older adult perceptions of the physicians' role in promoting physical activity. Disabil Rehabil 2013 Jul;35(14):1191-1198. [doi: 10.3109/09638288.2012.726314] [Medline: 23072684]

27. Blackwell DL, Lucas JW, Clarke TC. Summary health statistics for US adults: National health interview survey, 2012. Vital Health Stat 102014 Feb(260):1-161 [FREE Full text] [Medline: 24819891]

28. Forjuoh SN, Lee C, Wang S, Hong Y, Ory MG. Patient-physician discussion of physical activity and environmental barriers. Prev Med 2011 Sep;53(3):209-210. [doi: 10.1016/j.ypmed.2011.07.010] [Medline: 21803071]

29. Forjuoh S, Lee C, Wang S, Hong Y, Ory MG. Family physicians' perception on environmental influences in promoting physical activity in their obese patients. Open J Prev Med 2012 Nov;2(4):423-428. [doi: 10.4236/ojpm.2012.24060]

30. Hong Y, Ory MG, Lee C, Wang S, Pulczinksi J, Forjuoh SN. Walking and neighborhood environments for obese and overweight patients: Perspectives from family physicians. Fam Med 2012 May;44(5):336-341 [FREE Full text] [Medline: 23027116]

31. Forjuoh SN, Ory MG, Wang S, des Bordes JK, Hong YA. Using the iPod Touch for patient health behavior assessment and health promotion in primary care. JMIR Mhealth Uhealth 2014 Mar 21;2(1):e14 [FREE Full text] [doi: 10.2196/mhealth.2927] [Medline: 25100308]

32. Hong Y, Dahlke D, Ory M, Hochhalter A, Reynolds J, Purcell N, et al. Designing iCanFit: A mobile-enabled Web application to promote physical activity for older cancer survivors. JMIR Res Protoc 2013 Feb 14;2(1):e12 [FREE Full text] [doi: 10.2196/resprot.2440] [Medline: 23612053] 
33. Hong Y, Goldberg D, Dahlke DV, Ory MG, Cargill JS, Coughlin R, et al. Testing usability and acceptability of a Web application to promote physical activity (iCanFit) among older adults. JMIR Hum Factors 2014 Oct 13;1(1):e2 [FREE Full text] [doi: 10.2196/humanfactors.3787] [Medline: 27025254]

34. Hong Y, Goldberg D, Ory M, Towne JS, Forjuoh S, Kellstedt D, et al. Efficacy of a mobile-enabled Web app (iCanFit) in promoting physical activity among older cancer survivors: A pilot study. JMIR Cancer 2015 Jun 26;1(1):e7 [FREE Full text] [doi: 10.2196/cancer.4389] [Medline: 28410158]

35. Diaz KM, Krupka DJ, Chang MJ, Peacock J, Ma Y, Goldsmith J, et al. Fitbit@: An accurate and reliable device for wireless physical activity tracking. Int J Cardiol 2015 Apr 15;185:138-140 [FREE Full text] [doi: 10.1016/j.ijcard.2015.03.038] [Medline: 25795203]

36. Forjuoh S, Bolin J, Huber JJ, Vuong A, Adepoju O, Helduser J, et al. Behavioral and technological interventions targeting glycemic control in a racially/ethnically diverse population: A randomized controlled trial. BMC Public Health 2014 Jan 23;14:71 [FREE Full text] [doi: 10.1186/1471-2458-14-71] [Medline: 24450992]

37. Norman C, Skinner HA. eHEALS: The eHealth Literacy Scale. J Med Internet Res 2006 Nov 14;8(4):e27 [FREE Full text] [doi: $10.2196 / j m i r .8 .4 . e 27]$ [Medline: $\underline{17213046]}$

38. Craig CL, Marshall AL, Sjöström M, Bauman AE, Booth ML, Ainsworth BE, et al. International physical activity questionnaire: 12-country reliability and validity. Med Sci Sports Exerc 2003 Aug;35(8):1381-1395. [doi: 10.1249/01.MSS.0000078924.61453.FB] [Medline: 12900694]

39. Ware J, Kosinski M, Keller SD. A 12-Item Short-Form Health Survey: Construction of scales and preliminary tests of reliability and validity. Med Care 1996 Mar;34(3):220-233. [Medline: 8628042]

40. Epstein RM, Franks P, Fiscella K, Shields CG, Meldrum SC, Kravitz RL, et al. Measuring patient-centered communication in patient-physician consultations: Theoretical and practical issues. Soc Sci Med 2005 Oct;61(7):1516-1528. [doi: 10.1016/j.socscimed.2005.02.001] [Medline: 16005784]

41. Schmittdiel J, Mosen DM, Glasgow RE, Hibbard J, Remmers C, Bellows J. Patient Assessment of Chronic Illness Care (PACIC) and improved patient-centered outcomes for chronic conditions. J Gen Intern Med 2008 Jan;23(1):77-80 [FREE Full text] [doi: 10.1007/s11606-007-0452-5] [Medline: 18030539]

42. Sallis JF, Grossman RM, Pinski RB, Patterson TL, Nader PR. The development of scales to measure social support for diet and exercise behaviors. Prev Med 1987 Nov;16(6):825-836. [Medline: 3432232]

43. Cadmus-Bertram LA, Marcus BH, Patterson RE, Parker BA, Morey BL. Randomized trial of a Fitbit-based physical activity intervention for women. Am J Prev Med 2015 Sep;49(3):414-418 [FREE Full text] [doi: 10.1016/j.amepre.2015.01.020] [Medline: 26071863]

44. Cohen J. Statistical Power Analysis for the Behavioral Sciences. 2nd edition. Hillsdale, NJ: Lawrence Erlbaum Associates; 1988.

45. Donner A, Birkett N, Buck C. Randomization by cluster. Sample size requirements and analysis. Am J Epidemiol 1981 Dec;114(6):906-914. [Medline: 7315838]

46. Laird N, Ware JH. Random-effects models for longitudinal data. Biometrics 1982 Dec;38(4):963-974. [Medline: 7168798]

47. Byrne BM. Structural Equation Modeling With Mplus: Basic Concepts, Applications, and Programming. New York, NY: Routledge; 2012.

48. Geiser C. Data Analysis With Mplus. New York, NY: The Guilford Press; 2013.

49. Glasgow RE, Strycker LA, Kurz D, Faber A, Bell H, Dickman JM, et al. Recruitment for an Internet-based diabetes self-management program: Scientific and ethical implications. Ann Behav Med 2010 Aug;40(1):40-48. [doi: 10.1007/s12160-010-9189-1] [Medline: 20411443]

50. Takacs J, Pollock CL, Guenther JR, Bahar M, Napier C, Hunt MA. Validation of the Fitbit One activity monitor device during treadmill walking. J Sci Med Sport 2014 Sep;17(5):496-500. [doi: 10.1016/j.jsams.2013.10.241] [Medline: 24268570]

51. Dontje ML, de Groot M, Lengton RR, van der Schans CP, Krijnen WP. Measuring steps with the Fitbit activity tracker: An inter-device reliability study. J Med Eng Technol 2015;39(5):286-290. [doi: 10.3109/03091902.2015.1050125] [Medline: 26017748]

\section{Abbreviations}

BMI: body mass index

CAB: Community Advisory Board

E-HEALS: eHealth literacy scale

EHR: electronic health record

HBA: health behavior assessment

ICC: intraclass correlation coefficient

MVPA: moderate-to-vigorous physical activity

PACIC: Patient Assessment of Chronic Illness Care

PCP: primary care provider

PESCA: Program to Enhance Scholarly and Creative Activities 
RCT: randomized controlled trial

SEM: structural equation modeling

SMS: short message service

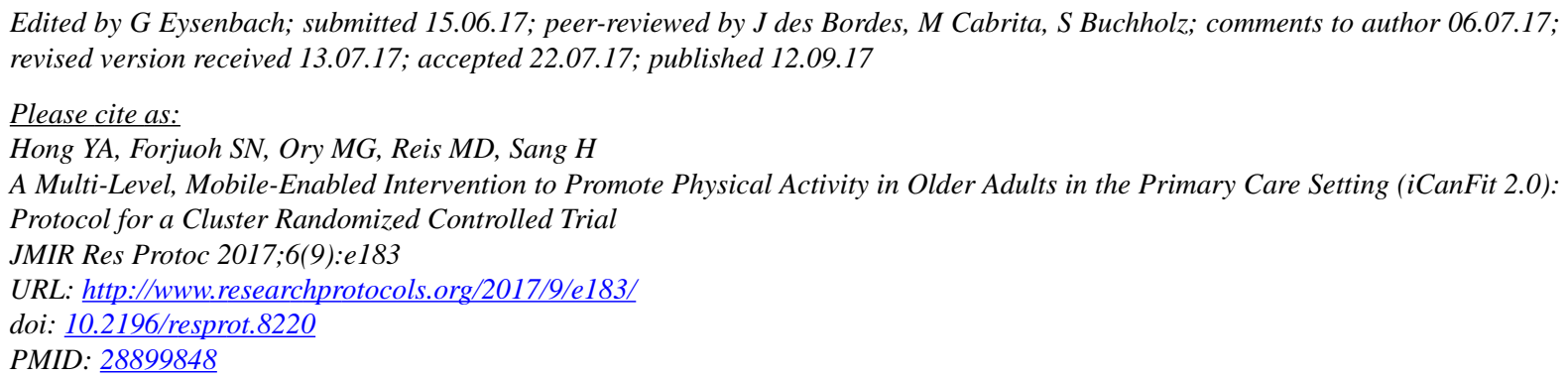

(CY Alicia Hong, Samuel N Forjuoh, Marcia G Ory, Michael D Reis, Huiyan Sang. Originally published in JMIR Research Protocols (http://www.researchprotocols.org), 12.09.2017. This is an open-access article distributed under the terms of the Creative Commons Attribution License (https://creativecommons.org/licenses/by/4.0/), which permits unrestricted use, distribution, and reproduction in any medium, provided the original work, first published in JMIR Research Protocols, is properly cited. The complete bibliographic information, a link to the original publication on http://www.researchprotocols.org, as well as this copyright and license information must be included. 